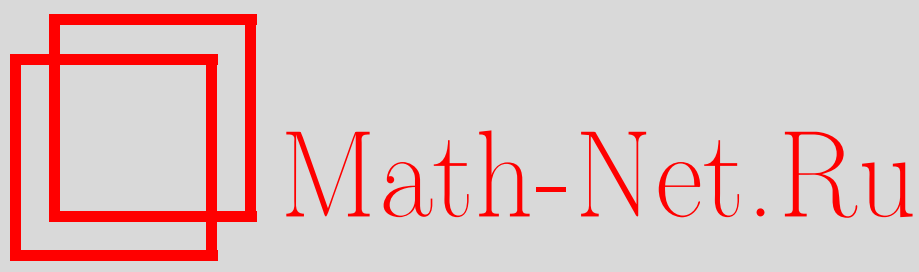

Д. Н. Иванов, Ортогональные разложения ассоциативных алгебр и сбалансированные системы идемпотентов, Матем. сб., 1998, том 189, номер 12, 83-102

DOI: https://doi.org/10.4213/sm367

Использование Общероссийского математического портала Math-Net.Ru подразумевает, что вы прочитали и согласны с пользовательским соглашением http://www . mathnet.ru/rus/agreement

Параметры загрузки:

IP: 3.81 .55 .215

26 апреля 2023 г., 04:38:20 
УДК $512.538+512.542+519.1$

Д. Н. Иванов

\title{
Ортогональные разложения ассоциативных алгебр и сбалансированные системы идемпотентов
}

\author{
В статье доказьвается гипотеза о делимости для коммутативных ортогональ- \\ ных разложений. Кроме этого, рассматриваются адамаровы разложения и сба- \\ лансированные системы идемпотентов. \\ Библиографофия: 8 названий.
}

\section{Введение}

Пусть $\mathbf{A}$ - ассоциативная конечномерная над полем комплексных чисел $\mathbb{C}$ алгебра, $\operatorname{Tr}_{A}=\operatorname{Tr}$ - след регулярного правого (или левого) представления алгебры $\mathbf{A}, \operatorname{Tr}_{A} x y$ - билинейная симметричная инвариантная форма на $\mathbf{A}$, которую будем назьвать формой следа. Как известно, алгебра $\mathbf{A}$ полупроста тогда и только тогда, когда форма следа невырождена на А. В дальнейшем ограничимся рассмотрением полупростых алгебр. Таким образом, в силу теоремы Веддербарна А изоморфна конечной прямой сумме матричных алгебр $\mathrm{M}_{n}(\mathbb{C})=\mathrm{M}_{n}$. Напомним определение ортогонального разложения алгебры $\mathbf{A}$.

ОПРЕДЕЛЕНИЕ. Семейство собственных неединичных подалгебр $\mathscr{D}=\left\{\mathbf{B}_{i}, i \in I\right\}$ образует ортогональное разложение $(O P)$ алгебры $\mathbf{A}$, если

(1) все подалгебры $\mathbf{B}_{i}$ полупросты и содержат единичный элемент $\mathbf{1}_{A}$ алгебры A;

(2) алгебра $\mathbf{A}$ линейно порождается подалгебрами $\mathbf{B}_{i}$, т.е. $\mathbf{A}=\sum_{i} \mathbf{B}_{i}$;

(3) (условие ортогональности) ортогональные дополнения к $\mathbf{1}_{A}$ в каждой из подалгебр семейства $\mathscr{D}$ ортогональны между собой, т.е. если $x \in \mathbf{B}_{i}, y \in \mathbf{B}_{j}$, $i, j \in I, i \neq j$, то

$$
\operatorname{Tr} x=\operatorname{Tr} y=0 \Rightarrow \operatorname{Tr} x y=0 .
$$

ЗАмЕчАниЕ. (а) Условие полупростоты подалгебры $\mathbf{B}_{i}$ равносильно невырожденности ограничения на эту подалгебру формы следа. Поэтому из условия ортогональности вытекает, что каждые две различные подалгебры из $\mathscr{D}$ пересекаются по единичной подалгебре $\left\langle\mathbf{1}_{A}\right\rangle$.

(b) На практике для проверки условия ортогональности часто бывает удобно использовать его эквивалентную формулировку: если $x \in \mathbf{B}_{i}, y \in \mathbf{B}_{j}, i, j \in I$, $i \neq j$, то

$$
\frac{\operatorname{Tr} x}{n} \cdot \frac{\operatorname{Tr} y}{n}=\frac{\operatorname{Tr} x y}{n},
$$

где $n=\operatorname{Tr} \mathbf{1}_{A}=\operatorname{dim} \mathbf{A}$. 
Ортогональное разложение $\mathscr{D}$, все подалгебры которого коммутативны, будем называть коммутативнылм. Если все подалгебры в $\mathscr{D}$ изоморфны алгебре $\mathbf{B}$, то будем назьвать $\mathscr{D}$ однородным OP muпа В (или просто “OP типа В"). Через $k \mathrm{M}_{n}$ обозначим прямую сумму $k$ экземпляров алгебры $\mathrm{M}_{n}$.

Понятие ортогонального разложения объединяет такие известные темы, как конечные аффинные плоскости, классические матрицы Адамара, конечные расщепляемые группы, ортогональные разложения простых алгебр Ли типа $A_{n}$ в сумму картановских подалгебр (см. [1]). Именно:

1) конечная аффинная плоскость порядка $n$ соответствует однородному ОР типа $n \mathrm{M}_{1}$ коммутативной алгебры $n^{2} \mathrm{M}_{1}$;

2) матрица Адамара порядка $n$ соответствует однородному OP типа $2 \mathrm{M}_{1}$ алгебры $n \mathrm{M}_{1}$

3) если подгруппы $\left\{H_{i}\right\}$ расщепляют группу $G$, то подалгебры $\left\{\mathbb{C}\left[H_{i}\right]\right\}$ образуют ОР групповой алгебры $\mathbb{C}[G]$;

$4)$ ортогональному разложению простой алгебры Ли типа $A_{n}$ в сумму картановских подалгебр соответствует однородное ОР типа $(n+1) \mathrm{M}_{1}$ алгебры матриц $\mathrm{M}_{n+1}$.

Одной из фундаментальных проблем теории ортогональных разложений ассоциативных алгебр является

ГиПотеЗА 1 (гипотеза о делимости). Алгебра является свободным модулем (левым или правым) над каждой подалгеброй из семейства, образующего ее ортогональное разложение.

Название "гипотеза о делимости" объясняется тем, что, как показано в [2], алгебра $\mathbf{A}$ является свободньм модулем над полупростой подалгеброй $\mathbf{B}$, содержащей единичньй элемент $\mathbf{1}_{A}$, тогда и только тогда, когда ограничение $\operatorname{Tr}_{A}$ на $\mathbf{B}$ пропорционально $\operatorname{Tr}_{B}$. Коэффициент пропорциональности в этом случае будет цельм числом, равньм отношению размерностей $\operatorname{dim} \mathbf{A} / \operatorname{dim} \mathbf{B}$.

С гипотезой о делимости связана (точнее является ее прямым следствием)

ГиПотЕЗА 2 (гипотеза об измельчении). Если подалгебры $\{\mathbf{B}, \mathbf{C}, \ldots, \mathbf{D}\}$ образуют ОР алгебры $\mathbf{A}$ и подалгебры $\left\{\mathbf{B}_{1}, \ldots, \mathbf{B}_{k}\right\}$ образуют ОР алгебры $\mathbf{B}$, то подалгебрьи $\left\{\mathbf{B}_{1}, \ldots, \mathbf{B}_{k}, \mathbf{C}, \ldots, \mathbf{D}\right\}$ образуют ОР алгебры $\mathbf{A}$.

Отметим, что доказательство гипотезы о делимости, приведенное в [2], оказалось ошибочным, о чем автору сообщили А. И. Кострикин и Фам Хыу Тьеп.

Основным результатом данной работы является

ТЕОРема 1. Гипотеза о делимости справедлива для коммутативных ортогональных разложсений.

Теорема 1 усиливает предыдуший результат автора о справедливости гипотезы 1 в случае однородных ОР коммутативных алгебр (см. [3]). Новое продвижение в гипотезе о делимости позволяет утверждать, что гипотеза об измельчении выполняется для коммутативных ОР. Изложению доказательства теоремы 1 посвящен первый параграфф статьи.

В $\S 2$ рассматриваются приложения теоремы 1 к однородным OP типа $2 \mathrm{M}_{1}-$ так называемым адамаровым разложсениям. Алгебры, допускающие адамаровы разложения, будем назьвать адамаровыми. В $\S 2$, в частности, доказывается теорема о тензорном произведении и о прямой сумме адамаровых алгебр. 
Также в $§ 2$ исследуются гипотеза о делимости размерности адамаровой алгебры на 4 и адамаровы разложения в алгебре матриц $\mathrm{M}_{n}$.

В $\S 3$ вводится понятие сбалансированных систем идемпотентов, которое обобщает комбинаторные блок-схемы. Исследуются связи сбалансированных систем с ортогональньги разложениями и кратно транзитивными группами.

Автор приносит благодарность А.И. Кострикину за поддержку и внимание к работе.

\section{§1. Гипотеза о делимости}

В этом параграфе доказывается центральная теорема данной статьи.

ТЕОРемА 1. Гипотеза 1 справедлива для коммутативных ортогональных разлоэсений.

ДокАЗАТЕЛЬство. Пусть алгебра $\mathbf{A} \cong \bigoplus_{p=1}^{q} \mathrm{M}_{n_{p}}$ допускает коммутативное $\mathrm{OP} \mathscr{D}=\left\{\mathbf{A}_{s}, s=\overline{1, r}\right\}$. Будем считать, что $\mathbf{A}$ реализована как блочно-диагональная подалгебра в алгебре матриц $\mathrm{M}_{d}$, где $d=\sum_{p=1}^{q} n_{p}$. Таким образом, $\mathbf{1}_{A}=E_{d}-$ единичная матрица порядка $d$.

Положим $n_{1}+\cdots+n_{p}=\sigma_{p}, p=\overline{1, q}$ (таким образом, $\left.\sigma_{q}=d\right)$, и если натуральное число $i$ таково, что $\sigma_{p-1}<i \leqslant \sigma_{p}$, то определим $n(i)=n_{p}$.

Как и раньше, $\operatorname{Tr}$ обозначает след регулярного представления алгебры А. Через $E_{i j}$ обозначим матричные единищы в $\mathrm{M}_{d}, X=\left(x_{i j}\right)$-матрица в $\mathrm{M}_{d}$ с коэффициентами $x_{i j}$, т.е. $X=\sum_{i, j=1}^{d} x_{i j} E_{i j}$.

Следующая лемма вытекает непосредственно из определений.

Лемма 1.1. (1) Ecли $X=\left(x_{i j}\right) \in \mathbf{A}$, mo $\operatorname{Tr} X=\sum_{i=1}^{d} n(i) x_{i i}$.

(2) $E_{i j} \in \mathbf{A} \Longleftrightarrow i, j \in\left(\sigma_{p-1}, \sigma_{p}\right]$ для некоторого $p$.

(3) $E_{i j} \in \mathbf{A} \Longleftrightarrow E_{j i} \in \mathbf{A} \Rightarrow n(i)=n(j)$.

(4) $\mathbf{1}_{A}=\sum_{i=1}^{d} E_{i i}$.

Пусть $\operatorname{Pr} \mathbf{A}_{s}=\left\{A_{s}, B_{s}, \ldots, C_{s}\right\}$ - множество примитивных идемпотентов подалгебры $\mathbf{A}_{s}, s=\overline{1, r}$ (количество элементов в $\operatorname{Pr} \mathbf{A}_{s}$ не меньше 2 и зависит от $s$ ). Положим $A_{s}=\left(a_{i j}(s)\right), B_{s}=\left(b_{i j}(s)\right), \ldots, C_{s}=\left(c_{i j}(s)\right), k_{s}=\operatorname{Tr} A_{s}, l_{s}=\operatorname{Tr} B_{s}$, $\ldots, m_{s}=\operatorname{Tr} C_{s}$.

Так как ограничение $\operatorname{Tr}$ на подалгебры $\left\langle\mathbf{1}_{A}\right\rangle, \mathbf{A}_{s}, s=\overline{1, r}$, невырожденно, то алгебра $\mathbf{A}$ является ортогональной прямой суммой подпространств $\left\langle\mathbf{1}_{A}\right\rangle$, $\mathbf{A}_{s}^{\circ}=\left\{X \in \mathbf{A}_{s}: \operatorname{Tr} X=0\right\}, s=\overline{1, r}$. Отсюда следуют две леммы.

ЛЕмма 1.2. Элементы $\mathbf{1}_{A}, B_{s}, \ldots, C_{s}, s=\overline{1, r}$, линейно независимы и образуют базис алгебры $\mathbf{A .}$

ЛЕмма 1.3 (тождество ортогональности). Для любого $X \in \mathbf{A}$ имеет место равенство

$$
\operatorname{Tr} X^{2}+\frac{r-1}{n}(\operatorname{Tr} X)^{2}=\sum_{s=1}^{r}\left(\frac{\left(\operatorname{Tr} X A_{s}\right)^{2}}{k_{s}}+\frac{\left(\operatorname{Tr} X B_{s}\right)^{2}}{l_{s}}+\cdots+\frac{\left(\operatorname{Tr} X C_{s}\right)^{2}}{m_{s}}\right),
$$

əде $n=\operatorname{Tr} \mathbf{1}_{A}=\operatorname{dim} \mathbf{A}=\sum_{p=1}^{q} n_{p}^{2}$.

Тождество ортогональности (1.1) будет основньм инструментом в доказательстве теоремы 1. Нам понадобится также следующее утверждение из работы [2]. 
Лемма 1.4. Алгебра $\mathbf{A}$ является свободным $\mathbf{A}_{s}$-модулем тогда и только тогда, когда ограничение $\operatorname{Tr}_{A}$ на $\mathbf{A}_{s}$ пропориионально $\operatorname{Tr}_{A_{s}}$.

Так как значения $\operatorname{Tr}_{A_{s}}$ на примитивных идемпотентах подалгебры $\mathbf{A}_{s}$ все равны 1 , то для доказательства теоремы 1 в силу леммы 1.4 достаточно показать, что $k_{s}=l_{s}=\cdots=m_{s}$ при любом $s=\overline{1, r}$.

Основная идея доказательства теоремы 1 заключается в выводе из тождества ортогональности (1.1) следующего равенства:

$$
\left(1+\frac{r-1}{n}\right) \cdot \mathbf{1}_{A}=\sum_{s=1}^{r}\left(\frac{A_{s}}{k_{s}}+\frac{B_{s}}{l_{s}}+\cdots+\frac{C_{s}}{m_{s}}\right)
$$

Теперь, вычитая из обеих частей равенства (1.2)

$$
\sum_{s=1}^{r}\left(\frac{A_{s}}{k_{s}}+\frac{B_{s}}{k_{s}}+\cdots+\frac{C_{s}}{k_{s}}\right)=\left(\sum_{s=1}^{r} \frac{1}{k_{s}}\right) \cdot \mathbf{1}_{A}
$$

получаем

$$
\left(1+\frac{r-1}{n}-\sum_{s=1}^{r} \frac{1}{k_{s}}\right) \cdot \mathbf{1}_{A}=\sum_{s=1}^{r}\left(\left(\frac{1}{l_{s}}-\frac{1}{k_{s}}\right) B_{s}+\cdots+\left(\frac{1}{m_{s}}-\frac{1}{k_{s}}\right) C_{s}\right) .
$$

В силу леммы 1.2 матрицы $\mathbf{1}_{A}=E_{d}, B_{s}, \ldots, C_{s}, s=\overline{1, r}$, линейно независимы. Значит, $k_{s}=l_{s}=\cdots=m_{s}$.

Вывод равенства (1.2) из тождества ортогональности осушествим в следующей последовательности шагов.

Шаг 1. Положим в (1.1) $X=E_{i j} \in \mathbf{A}, i \neq j$. Тогда $\operatorname{Tr} X A_{s}=n(j) a_{i j}(s)$, $\operatorname{Tr} X B_{s}=n(j) b_{i j}(s), \ldots, \operatorname{Tr} X C_{s}=n(j) c_{i j}(s), \operatorname{Tr} X=\operatorname{Tr} X^{2}=0$. В результате получаем

$$
0=\sum_{s=1}^{r}\left(\frac{n^{2}(j) a_{i j}^{2}(s)}{k_{s}}+\frac{n^{2}(j) b_{i j}^{2}(s)}{l_{s}}+\cdots+\frac{n^{2}(j) c_{i j}^{2}(s)}{m_{s}}\right)
$$

Отсюда следует, что

$$
0=\sum_{s=1}^{r}\left(\frac{a_{i j}^{2}(s)}{k_{s}}+\frac{b_{i j}^{2}(s)}{l_{s}}+\cdots+\frac{c_{i j}^{2}(s)}{m_{s}}\right)
$$

Если $E_{i j} \notin \mathbf{A}$, то $a_{i j}(s)=b_{i j}(s)=\cdots=c_{i j}(s)=0$. Таким образом, (1.4) выполняется при любых $i \neq j$.

Шаг 2. Положим в (1.1) $X=E_{i j}+E_{j i} \in \mathbf{A}, i \neq j$. Тогда $\operatorname{Tr} X=0, \operatorname{Tr} X^{2}=$ $\operatorname{Tr}\left(E_{i i}+E_{j j}\right)=n(i)+n(j)$ и в результате имеем

$$
n(i)+n(j)=\sum_{s=1}^{r}\left(\frac{\left(n(j) a_{i j}(s)+n(i) a_{j i}(s)\right)^{2}}{k_{s}}+\cdots+\frac{\left(n(j) c_{i j}(s)+n(i) c_{j i}(s)\right)^{2}}{m_{s}}\right) .
$$


В силу (1.4) и леммы 1.1 (3) отсюда получаем

$$
\frac{1}{n(i)}=\frac{1}{n(j)}=\frac{1}{2}\left(\frac{1}{n(i)}+\frac{1}{n(j)}\right)=\sum_{s=1}^{r}\left(\frac{a_{i j}(s) a_{j i}(s)}{k_{s}}+\cdots+\frac{c_{i j}(s) c_{j i}(s)}{m_{s}}\right)
$$

если $E_{i j} \in \mathbf{A}$ и $i \neq j$.

Шаг 3. Так как $A_{s}^{2}=A_{s}$, то

$$
a_{i i}(s)=a_{i i}^{2}(s)+\sum_{t \neq i} a_{i t}(s) a_{t i}(s)=a_{i i}^{2}(s)+\sum_{t \neq i, E_{t i} \in \mathbf{A}} a_{i t}(s) a_{t i}(s) .
$$

Поделим это равенство на $k_{s}$ и просуммируем аналогичные равенства для $b_{i i}(s), \ldots, c_{i i}(s)$. Затем, учитьвая, что сушествует ровно $n(i)-1$ индексов $t$ таких, что $t \neq i, E_{t i} \in \mathbf{A}$, и равенство (1.5), суммируем по $s$ и в результате получаем

$$
\sum_{s=1}^{r}\left(\frac{a_{i i}(s)}{k_{s}}+\cdots+\frac{c_{i i}(s)}{m_{s}}\right)=\sum_{s=1}^{r}\left(\frac{a_{i i}^{2}(s)}{k_{s}}+\cdots+\frac{c_{i i}^{2}(s)}{m_{s}}\right)+\frac{n(i)-1}{n(i)} .
$$

Шаг 4. Положим в (1.1) $X=E_{i i}$. Тогда имеем

$$
n(i)+\frac{r-1}{n} \cdot n^{2}(i)=\sum_{s=1}^{r}\left(\frac{n^{2}(i) a_{i i}^{2}(s)}{k_{s}}+\frac{n^{2}(i) b_{i i}^{2}(s)}{l_{s}}+\cdots+\frac{n^{2}(i) c_{i i}^{2}(s)}{m_{s}}\right) .
$$

Отсюда

$$
\frac{1}{n(i)}+\frac{r-1}{n}=\sum_{s=1}^{r}\left(\frac{a_{i i}^{2}(s)}{k_{s}}+\frac{b_{i i}^{2}(s)}{l_{s}}+\cdots+\frac{c_{i i}^{2}(s)}{m_{s}}\right) .
$$

Шаг 5. Из (1.6) и (1.7) следует, что

$$
\sum_{s=1}^{r}\left(\frac{a_{i i}(s)}{k_{s}}+\frac{b_{i i}(s)}{l_{s}}+\cdots+\frac{c_{i i}(s)}{m_{s}}\right)=\frac{1}{n(i)}+\frac{r-1}{n}+\frac{n(i)-1}{n(i)}=1+\frac{r-1}{n}
$$

Таким образом, равенство (1.2) доказано для диагональных элементов. Осталось проверить его для недиагональных элементов.

Шаг 6. Положим в (1.1) $X=E_{j i}+E_{v u} \in \mathbf{A}$, где $i \neq j, u \neq v$ и среди индексов $i, j, u, v$, по крайней мере, три различных. Тогда $\operatorname{Tr} X=\operatorname{Tr} X^{2}=0$ и

$$
0=\sum_{s=1}^{r}\left(\frac{\left(n(j) a_{i j}(s)+n(v) a_{u v}(s)\right)^{2}}{k_{s}}+\cdots+\frac{\left(n(j) c_{i j}(s)+n(v) c_{u v}(s)\right)^{2}}{m_{s}}\right) .
$$

Отсюда в силу (1.4), после деления на $2 n^{2}(j) \cdot n^{2}(v)$ получаем

$$
0=\sum_{s=1}^{r}\left(\frac{a_{i j}(s) a_{u v}(s)}{k_{s}}+\frac{b_{i j}(s) b_{u v}(s)}{l_{s}}+\cdots+\frac{c_{i j}(s) c_{u v}(s)}{m_{s}}\right)
$$

Ясно, что ограничение $E_{j i}+E_{v u} \in \mathbf{A}$ в (1.9) можно снять. 
Шаг 7. Так как $A_{s}^{2}=A_{s}$, то при $i \neq j$ имеем

$$
a_{i j}(s)=\left(a_{i i}(s)+a_{j j}(s)\right) a_{i j}(s)+\sum_{t \neq i, j} a_{i t}(s) a_{t j}(s) .
$$

Поделим это равенство на $k_{s}$ и просуммируем аналогичные равенства для $b_{i j}(s), \ldots, c_{i j}(s)$. Затем суммируем по $s$ и, учитывая $(1.9)$, в результате получаем

$$
\begin{aligned}
& \sum_{s=1}^{r}(\left.\frac{a_{i j}(s)}{k_{s}}+\frac{b_{i j}(s)}{l_{s}}+\cdots+\frac{c_{i j}(s)}{m_{s}}\right) \\
&=\sum_{s=1}^{r}\left(\frac{\left(a_{i i}(s)+a_{j j}(s)\right) a_{i j}(s)}{k_{s}}+\frac{\left(b_{i i}(s)+b_{j j}(s)\right) b_{i j}(s)}{l_{s}}\right. \\
&\left.+\cdots+\frac{\left(c_{i i}(s)+c_{j j}(s)\right) c_{i j}(s)}{m_{s}}\right)
\end{aligned}
$$

при $i \neq j$.

Шаг 8. Положим в (1.1) $X=E_{i i}-E_{j j}$. Тогда $\operatorname{Tr} X=0, \operatorname{Tr} X^{2}=n(i)+n(j)$ и

$$
n(i)+n(j)=\sum_{s=1}^{r}\left(\frac{\left(n(i) a_{i i}(s)-n(j) a_{j j}(s)\right)^{2}}{k_{s}}+\cdots+\frac{\left(n(i) c_{i i}(s)-n(j) c_{j j}(s)\right)^{2}}{m_{s}}\right) .
$$

Шаг 9. Положим в (1.1) $X=E_{i i}-E_{j j}+E_{v u} \in \mathbf{A}, i \neq j, u \neq v$. Тогда $\operatorname{Tr} X=0$, $\operatorname{Tr} X^{2}=n(i)+n(j)$ и

$$
\begin{aligned}
n(i)+n(j)=\sum_{s=1}^{r}( & \frac{\left(n(i) a_{i i}(s)-n(j) a_{j j}(s)+n(v) a_{u v}(s)\right)^{2}}{k_{s}} \\
& \left.+\cdots+\frac{\left(n(i) c_{i i}(s)-n(j) c_{j j}(s)+n(v) c_{u v}(s)\right)^{2}}{m_{s}}\right) \\
=\sum_{s=1}^{r}\left(\frac{\left(n(i) a_{i i}(s)-n(j) a_{j j}(s)\right)^{2}}{k_{s}}+\cdots\right)+n^{2}(v) \sum_{s=1}^{r}\left(\frac{a_{u v}^{2}(s)}{k_{s}}+\cdots\right) & \\
& +2 n(v) \sum_{s=1}^{r}\left(\frac{\left(n(i) a_{i i}(s)-n(j) a_{j j}(s)\right) \cdot a_{u v}(s)}{k_{s}}+\cdots\right) .
\end{aligned}
$$

Учитывая (1.11) и (1.4), получаем

$$
n(i) \sum_{s=1}^{r}\left(\frac{a_{i i}(s) a_{u v}(s)}{k_{s}}+\cdots\right)=n(j) \sum_{s=1}^{r}\left(\frac{a_{j j}(s) a_{u v}(s)}{k_{s}}+\cdots\right) .
$$

Ясно, что условие $E_{u v} \in \mathbf{A}$ в (1.12) можно опустить. 
Шаг 10. Из (1.10) и (1.12) следует, что при $i \neq j$

$$
\begin{aligned}
\sum_{s=1}^{r} & \left(\frac{a_{i j}(s)}{k_{s}}+\frac{b_{i j}(s)}{l_{s}}+\cdots+\frac{c_{i j}(s)}{m_{s}}\right) \\
& =\left(1+\frac{n(i)}{n(j)}\right) \cdot \sum_{s=1}^{r}\left(\frac{a_{i i}(s) a_{i j}(s)}{k_{s}}+\cdots+\frac{c_{i i}(s) c_{i j}(s)}{m_{s}}\right) \\
& =\left(1+\frac{n(i)}{n(j)}\right) \cdot \frac{n(t)}{n(i)} \cdot \sum_{s=1}^{r}\left(\frac{a_{t t}(s) a_{i j}(s)}{k_{s}}+\cdots\right) \\
& =\left(\frac{1}{n(i)}+\frac{1}{n(j)}\right) \cdot \sum_{s=1}^{r}\left(\frac{n(t) a_{t t}(s) a_{i j}(s)}{k_{s}}+\cdots\right) .
\end{aligned}
$$

Суммируем по $t$, учитывая, что $\sum_{t=1}^{d} n(t) a_{t t}(s)=\operatorname{Tr} A_{s}=k_{s}$, и аналогичные соотношения для других идемпотентов,

$$
\begin{aligned}
d \cdot \sum_{s=1}^{r} & \left(\frac{a_{i j}(s)}{k_{s}}+\frac{b_{i j}(s)}{l_{s}}+\cdots+\frac{c_{i j}(s)}{m_{s}}\right) \\
= & \left(\frac{1}{n(i)}+\frac{1}{n(j)}\right) \\
& \times \sum_{s=1}^{r}\left(\left(\sum_{t=1}^{d} n(t) a_{t t}(s)\right) \cdot \frac{a_{i j}(s)}{k_{s}}+\cdots+\left(\sum_{t=1}^{d} n(t) c_{t t}(s)\right) \cdot \frac{c_{i j}(s)}{m_{s}}\right) \\
= & \left(\frac{1}{n(i)}+\frac{1}{n(j)}\right) \cdot \sum_{s=1}^{r}\left(a_{i j}(s)+b_{i j}(s)+\cdots+c_{i j}(s)\right)=0
\end{aligned}
$$

(последнее равенство вытекает из того, что $A_{s}+B_{s}+\cdots+C_{s}=E_{d}$ ). Значит,

$$
\sum_{s=1}^{r}\left(\frac{a_{i j}(s)}{k_{s}}+\frac{b_{i j}(s)}{l_{s}}+\cdots+\frac{c_{i j}(s)}{m_{s}}\right)=0
$$

и равенство (1.2) проверено для недиагональных элементов. Это завершает доказательство теоремы 1.

В качестве немедленного следствия теоремы 1 получаем подтверждение гипотезы об измельчении для коммутативных ортогональных разложений.

СлеДСТВИЕ 1. Пусть коммутативные подалгебры $\left\{\mathbf{B}, \mathbf{C}_{1}, \ldots, \mathbf{C}_{r}\right\}$ образуют $O P$ алгебри $\mathbf{A}$ и подалгебри $\left\{\mathbf{B}_{1}, \ldots, \mathbf{B}_{k}\right\}$ образуют $O P$ алгебры $\mathbf{B}$. Тогда подалгебры $\left\{\mathbf{B}_{1}, \ldots, \mathbf{B}_{k}, \mathbf{C}_{1}, \ldots, \mathbf{C}_{r}\right\}$ образуют (коммутативное) ОР алгебpbы $\mathbf{A}$.

ДоКАЗАТЕЛЬСТво. В силу теоремы 1 ограничение функции $\operatorname{Tr}_{A}$ на подалгебру $\mathbf{B}$ пропорционально функции $\operatorname{Tr}_{B}$. Поэтому если $x \in \mathbf{B}$, то

$$
\operatorname{Tr}_{A} x=0 \Longleftrightarrow \operatorname{Tr}_{B} x=0 .
$$

Так как условие ортогональности вьполняется для подалгебр $\mathbf{B}$ и $\mathbf{C}_{s}$, то в силу $(*)$ оно выполняется для подалгебр $\mathbf{B}_{i}$ и $\mathbf{C}_{s}, i=\overline{1, k}$. Также из $(*)$ вытекает выполнение условия ортогональности для подалгебр $\mathbf{B}_{i}, \mathbf{B}_{j}$. Следствие доказано. 


\section{§2. Адамаровы разложения и алгебры}

Как показано в [3], понятие матрицы Адамара порядка $n>2$ и однородного ортогонального разложения типа $2 \mathrm{M}_{1}$ коммутативной алгебры $n \mathrm{M}_{1}$ эквивалентны. Именно: неединичные строки нормализованной матрицы Адамара можно рассматривать как координаты $(n-1)$-й инволюции в базисе из примитивных идемпотентов алгебры $n \mathrm{M}_{1}$ и семейство подалгебр, порожденных этими инволюциями, образует однородное ОР типа $2 \mathrm{M}_{1}$. Это подсказывает следующее определение.

ОпРЕДЕЛЕНИЕ. Однородное ортогональное разложение типа $2 \mathrm{M}_{1}$ называется адамаровым разложением. Алгебра, допускающая адамарово разложение, называется адамаровой.

Теорема 1 и лемма 1.2, примененные к адамаровым разложениям, позволяют получить следующий важный результат.

ЛЕмма 2.1. Пусть подалгебры $\left\{\mathbf{B}_{1}, \ldots, \mathbf{B}_{r}\right\}$ образуют адамарово разложение алгебры $\mathbf{A}$. Пусть $\operatorname{Pr}\left(\mathbf{B}_{i}\right)=\left\{e_{i 1}, e_{i 2}\right\}-$ примитивные идемпотенты подалгебры $\mathbf{B}_{i}, i=\overline{1, r}$. Тогда

(1) число подалгебр в адамаровом разложении на единичу меньше размерности алгебры, т.е. $r=\operatorname{dim} \mathbf{A}-1$;

(2) $\operatorname{Tr}_{A} e_{i 1}=\operatorname{Tr}_{A} e_{i 2}=\frac{1}{2} \operatorname{dim} \mathbf{A}$ для любого $i=\overline{1, r}$, в частности, размерность адамаровой алгебры четна;

(3) в каждой подалгебре адамарова разложения содержится ровно две инволюиии с нулевым следом регулярного представления, причем эти инволюции, взятые по одной из всех подалгебр разложения, образуют базис пространства $\mathbf{A}^{\circ}=\left\{x \in \mathbf{A}: \operatorname{Tr}_{A} x=0\right\}$.

2.1. Прямые суммы и тензорные произведения адамаровых алгебр. Как известно [4], кронекерово произведение двух матриц Адамара будет матрицей Адамара. В наших терминах этот результат переформулируется следующим образом: тензорное произведение двух коммутативных адамаровых алгебр есть адамарова алгебра. Кроме этого, самая старая и знаменитая гипотеза о матрицах Адамара, утверждаюшая, что для всякого натурального $n$ кратного 4 , сушествует матрица Адамара порядка $n$, эквивалентна следуюшему утверждению: прямая сумма двух коммутативыш адамаровых алгебр есть адамарова алгебра. Зададимся вопросом: являются ли прямая сумма и тензорное произведение произвольных адамаровых алгебр адамаровыми алгебрами? Оказывается, что ответ на этот вопрос утвердительный в случае тензорного произведения. А в случае прямой суммы ответ будет утвердительным только при дополнительных ограничениях.

Теорема 2. Пусть $\mathbf{A}$ и $\mathbf{B}$ - адамаровы алгебры. Тогда

(1) тензорное произведение $\mathbf{A} \otimes \mathbf{B}-$ адамарова алгебра;

(2) если $\operatorname{dim} \mathbf{A}=\operatorname{dim} \mathbf{B}$, то прямая сумма $\mathbf{A} \oplus \mathbf{B}-$ адамарова алгебра.

ДоказАТЕЛЬство. Пусть семейства подалгебр $\left\{\mathbf{A}_{i}, i=\overline{1, n}\right\}$ и $\left\{\mathbf{B}_{j}, j=\overline{1, m}\right\}$ образуют адамаровы разложения алгебр $\mathbf{A}$ и $\mathbf{B}$, соответственно. Примитивные идемпотенты подалгебры $\mathbf{A}_{i}$ обозначим через $a_{i 1}$ и $a_{i 2}$, подалгебры $\mathbf{B}_{j}-$ через $b_{j 1}$ и $b_{j 2}$. По лемме 2.1

$$
\operatorname{Tr}_{A} a_{i 1}=\operatorname{Tr}_{A} a_{i 2}=\frac{1}{2} \operatorname{dim} \mathbf{A}, \quad \operatorname{Tr}_{B} b_{j 1}=\operatorname{Tr}_{B} b_{j 2}=\frac{1}{2} \operatorname{dim} \mathbf{B} .
$$


Из условия ортогональности имеем

$$
\operatorname{Tr}_{A} a_{i \varepsilon} a_{i^{\prime} \delta}=\frac{1}{4} \operatorname{dim} \mathbf{A}, \quad \operatorname{Tr}_{B} b_{j \varepsilon} b_{j^{\prime} \delta}=\frac{1}{4} \operatorname{dim} \mathbf{B}
$$

для $i \neq i^{\prime}, i, i^{\prime}=\overline{1, n}, j \neq j^{\prime}, j, j^{\prime}=\overline{1, m}, \varepsilon, \delta=1,2$.

(1) Рассмотрим следующие двумерные полупростые подалгебры в $\mathbf{A} \otimes \mathbf{B}$, содержащие $\mathbf{1}_{A \otimes B}$ :

$$
\begin{gathered}
\mathbf{C}_{j}=\left\langle\mathbf{1}_{A} \otimes b_{j 1}, \mathbf{1}_{A} \otimes b_{j 2}\right\rangle, \quad \mathbf{D}_{i}=\left\langle a_{i 1} \otimes \mathbf{1}_{B}, a_{i 2} \otimes \mathbf{1}_{B}\right\rangle \\
\mathbf{F}_{i j}=\left\langle a_{i 1} \otimes b_{j 1}+a_{i 2} \otimes b_{j 2}, a_{i 1} \otimes b_{j 2}+a_{i 2} \otimes b_{j 1}\right\rangle
\end{gathered}
$$

где $i=\overline{1, n}, j=\overline{1, m}$, а угловые скобки обозначают, что подалгебры порождены заключенными в скобки элементами. Используя (2.1) и (2.2), легко проверить, что для этих подалгебр выполняется условие ортогональности. Так как число указанных подалгебр равно

$$
n+m+n m=(n+1)(m+1)-1=\operatorname{dim} \mathbf{A} \cdot \operatorname{dim} \mathbf{B}-1=\operatorname{dim} \mathbf{A} \otimes \mathbf{B}-1,
$$

то они линейно порождают алгебру $\mathbf{A} \otimes \mathbf{B}$. Значит, семейство подалгебр

$$
\left\{\mathbf{C}_{j}, \mathbf{D}_{i}, \mathbf{F}_{i}, i=\overline{1, n}, j=\overline{1, m}\right\}
$$

образует адамарово разложение алгебры $\mathbf{A} \otimes \mathbf{B}$.

(2) Пусть $\operatorname{dim} \mathbf{A}=\operatorname{dim} \mathbf{B}$, т.е. $n=m$. Тогда, используя (2.1) и (2.2), легко показать, что подалгебры

$$
\begin{gathered}
\mathbf{C}_{i}=\left\langle\left(a_{i 1}, b_{i 1}\right),\left(a_{i 2}, b_{i 2}\right)\right\rangle, \quad \mathbf{D}_{i}=\left\langle\left(a_{i 1}, b_{i 2}\right),\left(a_{i 2}, b_{i 1}\right)\right\rangle, \\
\mathbf{E}=\left\langle\left(\mathbf{1}_{A}, 0\right),\left(0, \mathbf{1}_{B}\right)\right\rangle,
\end{gathered}
$$

где $i=\overline{1, n}$, удовлетворяют условию ортогональности. Так как их число равно

$$
2 n-1=\operatorname{dim} \mathbf{A} \oplus \mathbf{B}-1
$$

и все указанные подалгебры двумерны, полупросты и содержат единичный элемент $\mathbf{1}_{A \oplus B}$, то они образуют адамарово разложение алгебры $\mathbf{A} \oplus \mathbf{B}$. Теорема 2 доказана.

ПримеР. Рассмотрим алгебру $3 \mathrm{M}_{2}$. Как известно, алгебра $\mathrm{M}_{2}$ обладает единственным с точностью до сопряженности адамаровым разложением, соответствуюшим ортогональному разложению простой алгебры Ли типа $A_{1}$ в сумму картановских подалгебр [5], [1]. Например, в качестве трех попарно ортогональных инволюций, порождаюших подалгебры адамарова разложения, можно взять

$$
\alpha_{1}=\left(\begin{array}{cc}
1 & 0 \\
0 & -1
\end{array}\right), \quad \alpha_{2}=\left(\begin{array}{ll}
0 & 1 \\
1 & 0
\end{array}\right), \quad \alpha_{3}=\left(\begin{array}{cc}
0 & i \\
-i & 0
\end{array}\right) .
$$

В силу п. (2) теоремы 2 получаем, что алгебра $2 \mathrm{M}_{2}$ также адамарова. Таким образом, алгебра $3 \mathrm{M}_{2}$ является прямой суммой двух адамаровых алгебр. Однако справедлива 
Теорема 3. Алгебра $3 \mathrm{M}_{2}$ не является адамаровой.

Доказательство. Пусть $\mathbf{A}=3 \mathrm{M}_{2}=\left\{\left(X_{1}, X_{2}, X_{3}\right): X_{i} \in \mathrm{M}_{2}\right\}, E=E_{2}-$ единичная матрица матрица 2-го порядка,

$$
\left.\operatorname{Tr}_{A}\left(X_{1}, X_{2}, X_{3}\right)=2 \dot{(} \operatorname{tr} X_{1}+\operatorname{tr} X_{2}+\operatorname{tr} X_{3}\right),
$$

где $\operatorname{tr}$ - след матрицы. Пусть $\alpha=\left(A_{1}, A_{2}, A_{3}\right)$ - инволюция и $\operatorname{Tr}_{A} \alpha=0$. Тогда $\alpha$ может принадлежать к одному из следующих типов:

1-й тип: $\operatorname{tr} A_{1}=0, A_{2}=-A_{3}= \pm E$;

2-й тип: $\operatorname{tr} A_{2}=0, A_{1}=-A_{3}= \pm E$;

3-й тип: $\operatorname{tr} A_{3}=0, A_{1}=-A_{2}= \pm E$;

4-й тип: $\operatorname{tr} A_{1}=\operatorname{tr} A_{2}=\operatorname{tr} A_{3}=0$.

Заметим, что инволюции первых трех типов могут быть ортогональны, только если они одинакового типа. Например, в случае инволюций 1-го и 2-го типов имеем

$$
\operatorname{Tr}_{A}\left(\left(A_{1}, E,-E\right) \cdot\left(E, A_{2},-E\right)\right)=\operatorname{Tr}_{A}\left(A_{1}, A_{2}, E\right)=4 .
$$

Таким образом, все инволюции в подалгебрах адамарова разложения алгебры $\mathbf{A}$ принадлежат либо к одному из первых трех типов, например, к 1-му, либо к 4-му типу. Значит, эти инволюции лежат в подпространстве

$$
V=\left\{\left(X_{1}, X_{2}, X_{3}\right) \in \mathbf{A}: \operatorname{tr} X_{1}=0, \operatorname{tr} X_{2}+\operatorname{tr} X_{3}=0\right\}
$$

которое строго содержится в подпространстве $\mathbf{A}^{\circ}=\left\{X \in \mathbf{A}: \operatorname{Tr}_{A} X=0\right\}$. Это противоречит п. (3) леммы 2.1. Теорема 3 доказана.

ЗАмЕчАнИЕ. В [1] неточно указано, что вопрос о сушествовании ОР в алгебре $3 \mathrm{M}_{2}$ эквивалентен вопросу о сушествовании в этой алгебре адамарова разложения. В то время как последний вопрос решается в теореме 3 , первый-по-прежнему остается открытым. Наиболее вероятным представляется существование ОР, в котором одна подалгебра изоморфна $3 \mathrm{M}_{1}$ и является центром в $3 \mathrm{M}_{2}$, а остальные двумерны, причем порождаются инволюциями 4-го типа.

Подводя итог, отметим, что на сегодняшний день известно только два сравнительно общих условия на адамаровы алгебры $\mathbf{A}$ и $\mathbf{B}$, позволяющих утверждать, что прямая сумма $\mathbf{A} \oplus \mathbf{B}$ будет адамаровой алгеброй:

(1) (гипотетичное условие) коммутативность алгебр $\mathbf{A}$ и $\mathbf{B}$;

(2) (доказанное условие) равенство размерностей $\mathbf{A}$ и $\mathbf{B}$.

2.2. О делимости размерности адамаровой алгебры на 4. Теорема 1 позволяет утверждать, что размерность адамаровой алгебры четна. В некоторых случаях, например, если алгебра $\mathbf{A}$ коммутативна или проста, существование в $\mathbf{A}$ адамарова разложения влечет делимость $\operatorname{dim} \mathbf{A}$ на 4 . Так, для коммутативных алгебр это равносильно хорошо известному и легко доказываемому свойству делимости порядка $n \neq 2$ матрицы Адамара на 4 (см. [4]). А для простых алгебр $\mathbf{A}$ этот факт вытекает из того, что $\operatorname{dim} \mathbf{A}$ является квадратом натурального числа. По-видимому, одной из центральных проблем в теории адамаровых разложений станет 
ГИПОТЕЗА 3. Если алгебра адамарова, то ее размерность делится на 4.

В случае коммутативных алгебр предполагается, что справедливо также и обрашение гипотезы 3 . Однако в обшем случае, как показывает пример алгебры $3 \mathrm{M}_{2}$, это не так.

В качестве одного из подтверждений гипотезы 3 приведем следующий результат.

Теорема 4. Алгебра $\mathbf{A}=2 \mathrm{M}_{1} \oplus \mathrm{M}_{2 n_{1}} \oplus \cdots \oplus \mathrm{M}_{2 n_{k}}$ не является адамаровой.

ДоказАТЕЛьство. Пусть подалгебры $\mathbf{A}_{1}, \ldots, \mathbf{A}_{r}$ образуют адамарово разложение алгебры $\mathbf{A}, \operatorname{Pr}\left(\mathbf{A}_{i}\right)=\left\{E_{i 1}, E_{i 2}\right\}$ - примитивные идемпотенты в $\mathbf{A}_{i}$. Два примитивных идемпотента в $\mathbf{A}$ со следом 1 обозначим через $e_{1}$ и $e_{2}$. Положим $\mathbf{B}=\mathrm{M}_{2 n_{1}} \oplus \cdots \oplus \mathrm{M}_{2 n_{k}}$ и отождествим $\mathbf{B}$ с ее каноническим образом в $\mathbf{A}$, тогда $\mathbf{1}_{A}=e_{1}+e_{2}+\mathbf{1}_{B}-$ разложение единицы в сумму ортогональных идемпотентов.

По теореме 1 следы $\operatorname{Tr}_{A} E_{i 1}$ и $\operatorname{Tr}_{A} E_{i 2}$ равны и, значит, нечетны. Поэтому можно считать, что индексы идемпотентов $E_{i 1}, E_{i 2}$ подобраны таким образом, что для некоторых идемпотентов $f_{i 1}, f_{i 2} \in \mathbf{B}$ имеем $E_{i 1}=e_{1}+f_{i 1}, E_{i 2}=e_{2}+f_{i 2}$.

Разложим $e_{2}$ по базису $\mathbf{1}_{A}, e_{1}+f_{i 1}, i=\overline{1, r}$ :

$$
e_{2}=a \cdot \mathbf{1}_{A}+\sum_{i=1}^{r} b_{i} \cdot\left(e_{1}+f_{i 1}\right)
$$

Рассматривая проекции этого равенства на $e_{2}, e_{1}$ и $\mathbf{B}$, последовательно получаем

(1) $a=1$;

(2) $0=1+\sum_{i=1}^{r} b_{i}$;

(3) $0=\mathbf{1}_{B}+\sum_{i=1}^{r} b_{i} f_{i 1}$.

Так как $2 \cdot \operatorname{Tr}_{A} f_{i 1}=\operatorname{Tr}_{A} \mathbf{1}_{B}$, то (2) противоречит (3). Теорема 4 доказана.

В коммутативном случае алгебра, размерность которой не делится на 4 , не может содержать двух ортогональных инволюций с нулевым следом. В общем случае ситуация иная. Групповая алгебра группы диэдра $\mathbb{C}\left[D_{2 n+1}\right]$ содержит множество из $2 n+1$ попарно ортогональных инволюций с нулевьм следом, но ее размерность не делится на 4. Кроме того, в силу теоремы 4 эта алгебра неадамарова.

Отметим, что неизвестно, обладает ли адамаровым разложением прямая сумма двух матричных алгебр одинаковой нечетной размерности.

2.3. Адамаровы разложения в алгебрах матриц. Из теоремы 1 следует, что алгебра матриц нечетного порядка неадамарова. Довольно правдоподобной вьглядит

ГИПотЕЗА 4. Алгебра матрии четного порядка адамарова.

Гипотеза 4 равносильна утверждению о существовании в пространстве матриц четного порядка с нулевьм следом базиса из попарно ортогональных инволюций. Пока лучшим продвижением в этом направлении является 
Теорема 5. Пусть $\mathbf{A} \cong 2 n \mathrm{M}_{p}-$ блочно-диагональная подалгебра в $\mathrm{M}_{2 n p}$. Тогда ортогональное дополнение (относительно форми следа) $к \mathbf{A}$ в $\mathrm{M}_{2 n p}$ содержит базис из попарно ортогональных инволюций. $B$ частности, алгебра $\mathrm{M}_{2 n p}$ допускает ОР, в котором одна подалгебра изоморфна $2 n \mathrm{M}_{p}$, а остальные двумерныл. При этом получаем, что если алгебра $2 n \mathrm{M}_{p}$ адамарова, то $\mathrm{M}_{2 n p}$ - также адамарова алгебра.

Предпошлем доказательству теоремы 5 две леммы, в которых рассматриваются частные случаи $p=1$ и $n=1$.

Когда $p=1, \mathbf{A}$ - диагональная подалгебра и ортогональное дополнение к $\mathbf{A}$ пространство матриц с нулевой диагональю.

ЛЕмма 2.2. В пространстве матрии четного порядка с нулевой диагональю существует базис из попарно ортогональньх инволюций.

ДокаЗАТЕЛЬСтво. Пусть $E_{i j}$ - матричные единицы в $\mathrm{M}_{2 n}, E$ - единичная матрица порядка $2 n$. В доказательстве будем использовать 1-факторизацию полного графа $K_{2 n}$.

Рассмотрим множество пар $T=\{(1,2),(3,4), \ldots,(2 n-1,2 n)\}$, разбиваюшее множество чисел $\{1,2, \ldots, 2 n\}$. В терминах теории графов $T$ является 1 -фактором полного графа $K_{2 n}$. Две инволюции

$$
\alpha=\left(\begin{array}{ccccc}
0 & x_{1} & & & \\
x_{1}^{-1} & 0 & & & \\
& & \ddots & & \\
& & & 0 & x_{n} \\
& & & x_{n}^{-1} & 0
\end{array}\right), \quad \beta=\left(\begin{array}{ccccc}
0 & y_{1} & & & \\
y_{1}^{-1} & 0 & & & \\
& & \ddots & & \\
& & 0 & y_{n} \\
& & y_{n}^{-1} & 0
\end{array}\right),
$$

очеви дным образом связанные с 1-фактором $T$, ортогональны тогда и только тогда, когда

$$
\frac{x_{1}}{y_{1}}+\frac{y_{1}}{x_{1}}+\cdots+\frac{x_{n}}{y_{n}}+\frac{y_{n}}{x_{n}}=0 .
$$

Легко видеть, что существование $2 n$ попарно ортогональных инволюций вида $(* *)$ эквивалентно сушествованию квадратной матрицы $X=\left(x_{i j}\right)$ порядка $2 n$ такой, что

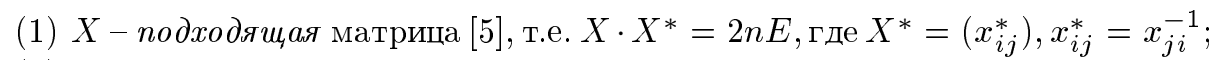

(2) строки матрицы $X$ разбиваются на пары взаимообратных, т.е. соответствующие компоненты строк в каждой паре - обратные числа.

Именно: матрицей $X$ с указанньми свойствами будет матрица, столбцы которой суть координаты $2 n$ попарно ортогональных инволюций вида $(* *)$ в базисе $E_{1,2}, E_{2,1}, \ldots, E_{2 n-1,2 n}, E_{2 n, 2 n-1}$.

Рассмотрим матрицу $C$ - таблицу комплексных неприводимых характеров циклической групшы порядка $2 n$. Ясно, что $C$ - подходящая матрица. Кроме этого, ее строки образуют циклическую группу порядка $2 n$ относительно покомпонентного умножения. Значит, только две строки матрицы $C$ обратны сами себе. С точностью до перестановки компонент это будут строки $(1,1, \ldots, 1)$ и $(1,-1,1,-1, \ldots, 1,-1)$. Заменим их на строки $(1, i, 1, i, \ldots, 1, i)$ и $(1,-i, 1,-i, \ldots, 1,-i)$. Так как новые строки лежат в подпространстве, 
порожденном удаленными строками, то новая матрица $C^{\prime}$ является подходящей и удовлетворяет условию (2).

Таким образом, используя матрицу $C^{\prime}$, по каждому 1-фактору можно построить $2 n$ попарно ортогональных инволюций. При этом инволюции, соответствующие непересекающимся 1-факторам, будут ортогональны. Для завершения доказательства леммы остается сослаться на существование $2 n-1$ попарно непересекающихся 1-фиакторов [6] (такие системы 1-факторов называются 1-факторизациями полного графа $\left.K_{2 n}\right)$.

ЛЕмма 2.3. Пусть $\mathbf{A} \cong \mathrm{M}_{p} \oplus \mathrm{M}_{p}$ - блочно-диагональная подалгебра в $\mathrm{M}_{2 p}$. Тогда ортогональное дополнение $\kappa \mathbf{A}$ в $\mathrm{M}_{2 p}$ содержит базис из попарно ортогональных инволюиий.

ДокАЗАТЕЛЬСтво. Рассмотрим матрицы $p$-го порядка

$$
P=\left(\begin{array}{ccccc}
1 & & & \\
& \varepsilon & & & \\
& \varepsilon^{2} & & \\
& & \ddots & \\
& & & \varepsilon^{p-1}
\end{array}\right), \quad D=\left(\begin{array}{ccccc}
0 & 0 & \ldots & 0 & 1 \\
1 & 0 & \ldots & 0 & 0 \\
0 & 1 & \ldots & 0 & 0 \\
\ldots & \ldots & \ldots & \ldots \\
0 & 0 & \ldots & 1 & 0
\end{array}\right)
$$

где $\varepsilon=e^{2 \pi i / p}$. Положим $U_{s t}=D^{s} P^{t}$. Тогда легко видеть, что матрицы $2 p$-го порядка

$$
\left(\begin{array}{cc}
0 & U_{s t} \\
U_{s t}^{-1} & 0
\end{array}\right), \quad\left(\begin{array}{cc}
0 & i U_{s t} \\
-i U_{s t}^{-1} & 0
\end{array}\right), \quad s, t=\overline{0, p-1},
$$

образуют искомый базис. Лемма доказана.

ДОКАЗАТЕЛЬСТво ТЕОРЕМЫ 5. Пусть $\left\{\alpha_{i}\right\}$ - базис из попарно ортогональных инволюций в пространстве матриц $2 n$-го порядка с нулевой диагональю, существование которого доказано в лемме $2.2,\left\{\beta_{j}\right\}$ - базис из попарно ортогональных инволюций, существование которого доказано в лемме 2.3. Тогда, рассматривая $\mathrm{M}_{2 n p}$ как тензорное произведение $\mathrm{M}_{2 n}$ и $\mathrm{M}_{p}$, получаем, что инволюции $\alpha_{i} \otimes \beta_{j}$ образуют искомый базис. Теорема 5 доказана.

ЗАмЕчАНИЕ. Отметим, что подходящие матрищы использовались ранее при конструировании ОР простых алгебр Ли типа $A_{n}$, а 1-фокторизации полного графа $K_{2 n}-$ при конструировании так назьваемых $E$-разложений простых алгебр Ли типа $D_{n}[7]$.

\section{§ 3. Сбалансированные системы идемпотентов}

\section{1. Определение, мотивировка и первые результаты.}

ОПРЕДЕЛЕНИЕ. Пусть $\mathscr{E}=\left\{e_{1}, \ldots, e_{v}\right\}$ - некоторое множество идемпотентов В алгебре А. Тогда

(1) $\mathscr{E}$ назьвается равноугольной $t$-системой (предполагаем $t<v$ ), если для каждого подмножества из $t$ идемпотентов $\left\{e_{i_{1}}, \ldots, e_{i_{t}}\right\}$ выполняется $\operatorname{Tr}_{A} e_{i_{1}} \cdots e_{i_{t}}=\lambda_{t}$ для некоторого фиксированного $\lambda_{t} \neq 0$;

(2) $\mathscr{E}$ называется максимальной $t$-системой, если $\mathscr{E}$ - равноугольная $t^{\prime}$-система для всех $t^{\prime} \leqslant t$, максимальная по включению; 
(3) равноугольная $t$-система $\mathscr{E}$ называется сбалансированной $t$-системой, если выполняется условие сбалансированности: $e_{1}+\cdots+e_{v}=k \cdot \mathbf{1}_{A}$;

(4) $\mathscr{E}$ называется полной $t$-системой, если $\mathscr{E}$ - равноугольная $t^{\prime}$-система для всех $t^{\prime} \leqslant t$, линейно порождающая алгебру $\mathbf{A}$.

Будем называть $\mathscr{E}$ порождающей системой, если идемпотенты из $\mathscr{E}$ порождают алгебру $\mathbf{A}$.

Обозначения этого определения сохраним на протяжении всего параграфа. Кроме этого, положим $\operatorname{dim} \mathbf{A}=b, \lambda_{1}=r, \lambda_{2}=\lambda$.

Следуюший пример является главной мотивировкой приведенного определения.

ПримеР. Напомним определение комбинаторной $t$-схемы [8]. Под $t$-схемой $c$ параметрами $\left(v, k, \lambda_{t}\right)$ понимается совокупность $\mathscr{B}$ подмножеств (называемых блоками) множества $\mathscr{V}$, состояшего из $v$ точек, такая, что

(1) каждое подмножество из $\mathscr{B}$ содержит $k$ точек;

(2) каждое подмножество из $t$ точек содержится ровно в $\lambda_{t}>0$ подмножествах из $\mathscr{B}$.

Матрица инцидентности $A t$-схемы, в которой строки отвечают точкам, а столбцы - блокам, имеет размеры $v \times b$, где $b$ - число блоков. Рассматривая строки матрицы $A$ как координаты элементов алгебры $b \mathrm{M}_{1}$ в базисе из примитивных идемпотентов этой алгебры, получаем сбалансированную $t$-систему идемпотентов в $b \mathrm{M}_{1}$. При этом условие сбалансированности вытекает из (1), а условие равноугольности из (2). Таким образом, комбинаторные $t$-схемы отвечают сбалансированным $t$-системам идемпотентов в коммутативных алгебрах.

Обозначения $v, k, \lambda, b, r$ являются стандартньми в комбинаторике, поэтому имеет смысл сохранить их для аналогичных величин в общей ситуации. Будем называть их параметрами соответствующих $t$-систем. Кроме того, $t$-систему, состоящую из $v$ идемпотентов со следом $r$, будем назьвать $t-(v, r)$ системой.

Лемма 3.1. Пусть $е, e_{1}, e_{2}$ - идемпотенты в алгебре А. Тогда

(i) $\operatorname{dim} \mathbf{A} e=\operatorname{dim} e \mathbf{A}=\operatorname{Tr}_{A} e$;

(ii) $\operatorname{dim} \mathbf{A}\left(e_{1}+e_{2}\right), \operatorname{dim}\left(e_{1}+e_{2}\right) \mathbf{A} \leqslant \operatorname{Tr}_{A} e_{1}+\operatorname{Tr}_{A} e_{2}$.

ДоказАТЕЛьство. Пункт (i) вытекает из разложения $\mathbf{A}=e \mathbf{A} \oplus e^{\prime} \mathbf{A}=$ $\mathbf{A} e \oplus \mathbf{A} e^{\prime}$, где $e^{\prime}=\mathbf{1}_{A}-e$, и равенств $e^{\prime} e=e e^{\prime}=0$.

Пункт (ii) следует из (i), так как, например, $\operatorname{dim}\left(e_{1}+e_{2}\right) \mathbf{A} \leqslant \operatorname{dim} e_{1} \mathbf{A}+\operatorname{dim} e_{2} \mathbf{A}$. Лемма доказана.

ЛЕмма 3.2. Пусть $\mathscr{E}=\left\{e_{1}, \ldots, e_{v}\right\}-$ сбалансированная 1-система в алгебpe A. Тогда

(i) $v r=k b$;

(ii) $k \geqslant 1$.

ДокАЗАТЕЛЬство. Применяя $\operatorname{Tr}_{A}$ к обеим частям равенства

$$
e_{1}+\cdots+e_{v}=k \cdot \mathbf{1}_{A},
$$

получаем (i). В силу леммы 3.1 из (3.1) следует

$$
\operatorname{dim} k \mathbf{1}_{A} \cdot \mathbf{A}=b \leqslant \operatorname{dim} e_{1} \mathbf{A}+\cdots+\operatorname{dim} e_{v} \mathbf{A}=\operatorname{Tr}_{A} e_{1}+\cdots+\operatorname{Tr}_{A} e_{v}=v r .
$$

Учитывая (i), отсюда выводим $k \geqslant 1$. Лемма доказана. 
Лемма 3.3. Пусть $\mathscr{E}=\left\{e_{1}, \ldots, e_{v}\right\}-$ сбалансированная 2-система в алгебpe A. Тогда

(i) $\mathscr{E}-$ сбалансированная 1-система;

(ii) $(v-1) \lambda=r(k-1) u k \neq 1$.

ДокАЗАТЕЛЬСтво. Домножая условие сбалансированности (3.1) справа на $e_{i}$, получаем $e_{1} e_{i}+\cdots+e_{i}^{2}+\cdots+e_{v} e_{i}=k \cdot e_{i}$. Отсюда следует, что

$$
e_{1} e_{i}+\cdots+e_{i-1} e_{i}+e_{i+1} e_{i}+\cdots+e_{v} e_{i}=(k-1) \cdot e_{i} .
$$

Применяя $\operatorname{Tr}_{A}$ к $(3.2)$, имеем $(v-1) \lambda=(k-1) \operatorname{Tr}_{A} e_{i}$. Так как $\lambda \neq 0$, то $k \neq 1$, и значит, $\operatorname{Tr}_{A} e_{i}=$ const $=r \neq 0$. Лемма доказана.

Лемма 3.4. Пусть $\mathscr{E}=\left\{e_{1}, \ldots, e_{v}\right\}-$ сбалансированная $t$-система в алгебpe $\mathbf{A} u t>2$. Тогда

(i) если $t=3$, то $\mathscr{E}-$ сбалансированная 2-система и $\lambda_{3}(v-2)=\lambda_{2}(k-2)$, при этом $k \neq 2$;

(ii) если алгебра $\mathbf{A}$ коммутативна, то $\mathscr{E}-$ сбалансированная $t^{\prime}$-система для всех $t^{\prime}<t u$

$$
\lambda_{t-1}(k-t+1)=\lambda_{t}(v-t+1)
$$

при этом $k \geqslant t$.

ДокаЗАТЕЛЬСтво. (i) Домножая (3.2) слева на $e_{j}, j \neq i$, и вычитая из обеих частей $e_{j} e_{i}$, получаем

$$
\sum_{s \neq j, i} e_{j} e_{s} e_{i}=(k-2) \cdot e_{j} e_{i}
$$

Применяя $\operatorname{Tr}_{A}$ к (3.3), имеем $\lambda_{3}(v-2)=(k-2) \operatorname{Tr}_{A} e_{j} e_{i}$. Так как $\lambda_{3} \neq 0$, то $k \neq 2$, и значит, $\operatorname{Tr}_{A} e_{j} e_{i}=$ const $=\lambda_{2} \neq 0$.

(ii) Достаточно доказать, что $\mathscr{E}-$ сбалансированная $(t-1)$-система. Пусть алгебра $\mathbf{A}$ коммутативна, тогда для $t-1$ различных идемпотентов имеем

$$
\begin{aligned}
e_{i_{1}} \cdots e_{i_{t-1}} & =\frac{1}{k} \cdot e_{i_{1}} \cdots e_{i_{t-1}}\left(e_{1}+\cdots+e_{v}\right) \\
& =\frac{t-1}{k} \cdot e_{i_{1}} \cdots e_{i_{t-1}}+\frac{1}{k} \cdot \sum_{s \neq i_{1}, \ldots, i_{t-1}} e_{i_{1}} \cdots e_{i_{t-1}} e_{s} .
\end{aligned}
$$

Применяя $\operatorname{Tr}_{A}$ к (3.4), получаем $(k-t+1) \operatorname{Tr}_{A} e_{i_{1}} \cdots e_{i_{t-1}}=\lambda_{t}(v-t+1)$. Так как $\lambda_{t} \neq 0$, то $k \neq t-1$ и $\operatorname{Tr}_{A} e_{i_{1}} \cdots e_{i_{t-1}}=\mathrm{const}=\lambda_{t-1} \neq 0$, т.е. $\mathscr{E}-$ сбалансированная $(t-1)$-система.

Неравенство $k \geqslant t$ следует из того, что $k$ - целое и $k \neq t-1, t-2, \ldots, 1$. Лемма доказана.

Следуюшая лемма содержит обобшение известного неравенства Фишера [4]. 
ЛЕмма 3.5. Пусть $\mathscr{E}=\left\{e_{1}, \ldots, e_{v}\right\}$ - сбалансированная 2 -система в алгебpe A. Тогда

(i) $r \neq \lambda$;

(ii) $r+\lambda(v-1) \neq 0$;

(iii) $\mathscr{E}$ - линейно независимая система, в частности, $v \leqslant b$.

ДокаЗАТЕЛЬство. (i) Если $r=\lambda$, то из леммы 3.3 (ii) следует, что $v=k$. Значит, в силу леммы 3.3 (i) и леммы 3.2 (i) получаем $r=b$. Так как $e_{i}$-идемпотенты, то из леммы 3.1 (i) вытекает, что $e_{1}=\cdots=e_{v}=\mathbf{1}_{A}$. Противоречие, так как $\mathscr{E}-$ система из, по крайней мере, двух различных идемпотентов.

(ii) Домножая условие сбалансированности (3.1) на $e_{i}$ (справа или слева) и применяя к получившемуся равенству $\operatorname{Tr}_{A}$, получаем $r+\lambda(v-1)=k r \neq 0$.

(iii) Вычисляя определитель Грама $G\left(e_{1}, \ldots, e_{v}\right)$, находим

$$
G\left(e_{1}, \ldots, e_{v}\right)=\operatorname{det}\left(\operatorname{Tr}_{A} e_{i} e_{j}\right)=\left|\begin{array}{cccc}
r & \lambda & \ldots & \lambda \\
\lambda & r & \ldots & \lambda \\
\ldots & \ldots & \ldots & \ldots \\
\lambda & \lambda & \ldots & r
\end{array}\right|=(r-\lambda)^{v-1}(r+\lambda(v-1))
$$

В силу доказанного в предыдущих пунктах $G\left(e_{1}, \ldots, e_{v}\right) \neq 0$. Значит, $\mathscr{E}-$ линейно независимая система. Лемма доказана.

ТЕОРЕма 6. Имеют место следующие включения классов 2-систем идемпотентов:

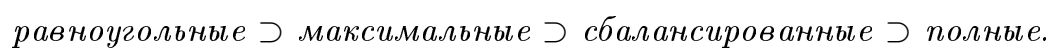

В частности, полная 2-система является базисом исходной алгебры.

ДокАЗАТЕльство. В доказательстве нуждаются только последние два включения.

1) Докажем включение максимальные つ сбалансированные.

Пусть $\mathscr{E}=\left\{e_{1}, \ldots, e_{v}\right\}$ - сбалансированная 2-система в алгебре А. Предположим, что $\mathscr{E}-$ не максимальна. Тогда существует идемпотент $e \in \mathbf{A}$ такой, что $\mathscr{E}^{\prime}=\mathscr{E} \cup\{e\}-$ равноугольная 2,1 -система. Домножив условие сбалансированности (3.1) для системы $\mathscr{E}$ на $е$ и применив к полученному равенству $\operatorname{Tr}_{A}$, получим $v \lambda=k r$. Так как в силу леммы 3.3 (ii) $(v-1) \lambda=r(k-1)$, то отсюда выводим $r=\lambda$. Это противоречит лемме 3.5 (i).

2) Докажем включение сбалансированные つ полные.

Пусть $\mathscr{E}$ - полная 2-система в алгебре $\mathbf{A}, \mathscr{E}^{\prime}$ - максимальная линейно независимая подсистема в $\mathscr{E}$. Тогда $\mathscr{E}^{\prime}$ - полная 2-система, являющаяся базисом в А. Легко видеть, что все коэффициенты в разложении $\mathbf{1}_{A}$ по базису $\mathscr{E}$ одинаковы. Значит, $\mathscr{E}^{\prime}$ - сбалансированная 2-система и в силу 1) $\mathscr{E}=\mathscr{E}$. Теорема доказана.

Полные 2-системы в коммутативных алгебрах отвечают симметричным 2-схемам. Последние занимают центральное место в комбинаторном анализе. Особенно актуальна проблема существования симметричных 2-схем с определенными параметрами. Отметим здесь известную теорему Брука-Райзера-Човлы [4]. Каких-либо общих результатов такого же характера для полных 2-систем в произвольных алгебрах пока не получено. Было бы интересно выяснить, какие из матричных алгебр допускают полные 2-системы идемпотентов. Вопрос этот открыт уже в случае $\mathrm{M}_{3}$. 
Отметим, что не существует полных $t$-систем при $t>2$. Так как если $\mathscr{E}=\left\{e_{1}, \ldots, e_{v}\right\}-$ полная 3 -система, то в разложении любого произведения $e_{i} e_{j}$ по базису $\mathscr{E}$ все коэффициенты будут одинаковы. Это значит, что $e_{i} e_{j}=\alpha \cdot \mathbf{1}_{A}$ для некоторого числа $\alpha \neq 0$. Таким образом, $e_{i}, e_{j}-$ обратимые элементы. Противоречие.

3.2. Ортогональные разложения и сбалансированные 2-системы. Если $\mathscr{D}$ - однородное $\mathrm{OP}$ коммутативной алгебры $v \mathrm{M}_{1}$, то в силу теоремы 1 все примитивные идемпотенты подалгебр из $\mathscr{D}$ имеют одинаковый след. В этом случае, как показано в [1], рассматривая примитивные идемпотенты алгебры А как точки, а примитивные идемпотенты подалгебр из $\mathscr{D}$ как блоки, разложению $\mathscr{D}$ отвечает 2 -схема ${ }^{1} \mathscr{S}$, которой, в свою очередь, согласно примеру п. 3.1 соответствует сбалансированная 2-система идемпотентов в алгебре $b \mathrm{M}_{1}$, где $b$ - число блоков в $\mathscr{S}$. Описанное соответствие удобно интерпретировать, используя матрицу инцидентности $A$ 2-схемы $\mathscr{S}$. Столбцы $A$ суть координаты примитивных идемпотентов подалгебр разложения $\mathscr{D}$ алгебры $v \mathrm{M}_{1}$, а строки $A$ - это координаты идемпотентов сбалансированной 2-системы в алгебре $b \mathrm{M}_{1}$.

Кроме указанного вьше, сушествуют иные способы связывать некоторые ортогональные разложения и сбалансированные 2-системы. Например, по адамарову разложению алгебры $4 n \mathrm{M}_{1}$ можно построить полную 2-систему (т.е. симметричную 2 -схему) в алгебре $(4 n-1) \mathrm{M}_{1}$. А однородному OP типа $n \mathrm{M}_{1}$ алгебры $n^{2} \mathrm{M}_{1}$ (это аффинная плоскость порядка $n$ ) соответствует полная 2-система в алгебре $\left(n^{2}+n+1\right) \mathrm{M}_{1}$ (проективная плоскость). Последний пример имеет также следующий аналог.

TЕОРема 7. Существует взаимнооднозначное соответствие между однородными ОР типа $n \mathrm{M}_{1}$ алгебры $\mathrm{M}_{n}$ и полными 2-системами идемпотентов со следом $n+1$ в алгебре $(n+1) \mathrm{M}_{1} \oplus \mathrm{M}_{n}$.

Нам понадобится

Лемма 3.6. Пусть $e_{1}, \ldots, e_{n}$ - примитивнъе идемпотенты в $\mathrm{M}_{n}$ такие, что $e_{1}+\cdots+e_{n}=E$, где $E-$ единичная матрица $n$-го поряджа. Тогда $e_{i} e_{j}=e_{j} e_{i}=0$ при $i \neq j$ и идемпотенты $e_{i}$ порождают подалгебру, изоморфную $n \mathrm{M}_{1}$.

ДоКАЗАТЕЛЬСТво. Воспользуемся двумя хорошо известными свойствами примитивных идемпотентов в $\mathrm{M}_{n}$ :

(a) $\operatorname{dim} e_{i} \mathrm{M}_{n}=n$;

(b) $\operatorname{dim} e_{i} \mathrm{M}_{n} e_{j}=1$.

Из этих свойств следует

$$
e_{i} \mathrm{M}_{n}=e_{i} \mathrm{M}_{n}\left(e_{1}+\cdots+e_{n}\right)=e_{i} \mathrm{M}_{n} e_{1} \oplus \cdots \oplus e_{i} \mathrm{M}_{n} e_{n}
$$

Так как $0=e_{i}\left(E-e_{i}\right)=e_{i} e_{1}+\cdots+e_{i} e_{i-1}+e_{i} e_{i+1}+\cdots+e_{i} e_{n}$, то из $(* * *)$ получаем $e_{i} e_{j}=0$. Лемма доказана.

\footnotetext{
${ }^{1}$ Если $\mathscr{D}$ - адамарово разложение, то получаем 3-схему.
} 
ДоКАЗАТЕЛЬСТВо ТЕОРЕМЫ 7. Положим $\mathbf{A}=(n+1) \mathrm{M}_{1} \oplus \mathrm{M}_{n}$ и будем отождествлять алгебры $(n+1) \mathrm{M}_{1}$ и $\mathrm{M}_{n}$ с их каноническими образами в А. Обозначим через $E$ и $F$ единичные элементы, соответственно, в $\mathrm{M}_{n}$ и в $(n+1) \mathrm{M}_{1}$.

Пусть подалгебры $H_{0}, H_{1}, \ldots, H_{n}$, изоморфные $n \mathrm{M}_{1}$, образуют однородное ОР алгебры $\mathrm{M}_{n}$ и $\operatorname{Pr}\left(H_{i}\right)=\left\{e_{i 1}, \ldots, e_{i n}\right\}$ - множество примитивных идемпотентов в $H_{i}$. Пусть $\operatorname{Pr}\left((n+1) \mathrm{M}_{1}\right)=\left\{f_{0}, f_{1}, \ldots, f_{n}\right\}$. Тогда легко видеть, что идемпотенты $F, e_{i j}+f_{i}, i=\overline{0, n}, j=\overline{1, n}$, образуют полную 2-систему.

Обратно, пусть $\mathscr{E}$ - полная 2-система идемпотентов со следом $(n+1)$ в А. Если $F \notin \mathscr{E}$, то, так как в силу условия сбалансированности каждый идемпотент $f_{i}$ должен являться слагаемым одного и того же количества идемпотентов из $\mathscr{E}$, получаем, что $n+1$ делит $n^{2}+n+1$. Противоречие показывает, что $F \in \mathscr{E}$. Тогда каждьй $f_{i}$ является слагаемьм ровно в $n$ идемпотентах из $\mathscr{E}: f_{i}+e_{i 1}, \ldots, f_{i}+e_{i n}$, где $e_{i j} \in \operatorname{Pr}\left(\mathrm{M}_{n}\right)$. Положим $E_{i}=e_{i 1}+\cdots+e_{i n}$. Тогда $\operatorname{Tr}_{A} E_{i} e_{s t}=\operatorname{Tr}_{A} E e_{s t}$ при всех $s, t$. Так как в силу полноты $\mathscr{E} e_{s t}$ линейно порождают $\mathrm{M}_{n}$, то в силу невырожденности формы следа на $\mathrm{M}_{n}$ получаем, что $E_{i}=E$. В силу леммы 3.6 отсюда следует, что подалгебры $H_{i}=\left\langle e_{i j}, j=\overline{1, n}\right\rangle_{\mathbb{C}}$ образуют однородное ОР типа $n \mathrm{M}_{1}$ алгебры $\mathrm{M}_{n}$. Теорема 7 доказана.

3.3. Сбалансированные $t$-системы и $t$-транзитивные группы подстановок. Пусть $G-t$-транзитивная группа подстановок степени $n, n>t \geqslant 2, G_{i}-$ стабилизатор точки, $i=1, \ldots, n$. Предположим, что в каждой из подгрупп $G_{i}$ выбрана подгруппа $H_{i}$ так, что

1) подгруппы $H_{1}, \ldots, H_{n}$ образуют $G$-орбиту относительно действия сопряжением;

2) для любых различных индексов $i_{1}, \ldots, i_{t}$ подмножества $H_{i_{1}} \cdots H_{i_{t}}=$ $\left\{h_{i_{1}} \cdots h_{i_{t}}: h_{i_{j}} \in H_{i_{j}}\right\}$ сопряжены в $G$.

Очевидно, эти условия вьполняются, если $H_{i}-$ характеристическая подгруппа в $G$.

Рассмотрим идемпотенты

$$
e_{i}=\frac{1}{h} \sum_{g \in H_{i}} g \in \mathbb{C}[G]
$$

где $h$ - порядок подгрупп $H_{i}$. Пусть $\varphi: G \rightarrow \mathrm{M}_{d}$ - неприводимое представление групшы $G$ с характером $\chi$ такое, что $H_{i} \nsubseteq \mathbb{\operatorname { k e r } \varphi}$ и

$$
\sum_{g \in H_{i}} \chi(g) \neq 0 .
$$

Тогда множество идемпотентов $\mathscr{E}=\left\{\varphi\left(e_{i}\right), i=1, \ldots, n\right\}$ в силу 2) является равноугольной $t$-системой, а в силу 1 ) удовлетворяет условию сбалансированности, т.е. $\mathscr{E}$ - сбалансированная $t$-система в $\mathrm{M}_{d}$.

Отметим, что если взять $H_{i}=G_{i}$, условия 1$)$ и 2) окажутся выполненными. Кроме того, подстановочный характер $\pi$ групп $G$ представляется в виде суммы $\pi=1_{G}+\chi$, где $1_{G}$ - единичньй, а $\chi$ - неприводимьй характеры групш $G$. Пусть $\varphi: G \rightarrow \mathrm{M}_{n-1}-$ представление, отвечающее $\chi$. Тогда получаем

$$
\sum_{g \in G_{i}} \pi(g)=2\left|G_{i}\right| \Longrightarrow \sum_{g \in G_{i}} \chi(g)=\left|G_{i}\right| .
$$


Значит, $\operatorname{tr} \varphi\left(e_{i}\right)=1$ и идемпотенты $\varphi\left(e_{i}\right), i=\overline{1, n}$, образуют сбалансированную $t-(n, n-1)$ систему в $\mathrm{M}_{n-1}$.

Рассматривая в качестве $G$ симметрическую группу $S_{n+1}$ в ее каноническом подстановочном представлении степени $n+1$, получаем, что справедлива

Tеорема 8. В $\mathrm{M}_{n}$ при $n \geqslant 2$ существует система идемпотентов, которая является сбалансированной $t-(n+1, n)$ системой при всех $t \leqslant n$.

ПримеРы. Сбалансированные $t$-системы в $\mathrm{M}_{2}$ и $\mathrm{M}_{3}$.

1) Из канонического подстановочного представления степени 3 симметрической групшы $S_{3}$, полагая $H_{i}=G_{i} \cong S_{2}$, получаем $2-(3,2)$ систему идемпотентов $\mathscr{E}_{1}$ в $\mathrm{M}_{2}$ :

$$
f_{1}=\frac{1}{2}\left(\begin{array}{ll}
1 & 1 \\
1 & 1
\end{array}\right), \quad f_{2}=\left(\begin{array}{rr}
1 & -\frac{1}{2} \\
0 & 0
\end{array}\right), \quad f_{3}=\left(\begin{array}{rr}
0 & 0 \\
-\frac{1}{2} & 1
\end{array}\right) .
$$

Приведенная система единственна с точностью до сопряженности в Aut $\mathrm{M}_{2}$ и имеет группу автоморфизмов Aut $\mathscr{E} 1 \cong S_{3}$.

2) Полная $2-(4,2)$ система $\mathscr{2} 2$ в $\mathrm{M}_{2}$ :

$$
f_{1}=\left(\begin{array}{ll}
1 & 0 \\
0 & 0
\end{array}\right), \quad f_{2}=\frac{1}{3}\left(\begin{array}{ll}
1 & 2 \\
1 & 2
\end{array}\right), \quad f_{3}=\frac{1}{3}\left(\begin{array}{cc}
1 & 2 \varepsilon^{2} \\
\varepsilon & 2
\end{array}\right), \quad f_{4}=\frac{1}{3}\left(\begin{array}{cc}
1 & 2 \varepsilon \\
\varepsilon^{2} & 2
\end{array}\right),
$$

где $\varepsilon=e^{2 \pi i / 3}$. Приведенная система единственна с точностью до сопряженности в Aut $\mathrm{M}_{2}$. Группа Aut $\mathscr{E}_{2}$ не может быть изоморфна $S_{4}$, так как, например, идемпотенты $f_{1}$ и $f_{2}$ порождают алгебру $\mathrm{M}_{2}$, и значит, Aut $\mathscr{E}_{2}$ не содержит транспозиции, переставляюшей $f_{3}$ и $f_{4}$. С другой стороны, Aut $\mathscr{E}_{2}$ содержит сопряжения матрицами

$$
X_{1}=\left(\begin{array}{cc}
1 & 2 \\
1 & -1
\end{array}\right), \quad X_{2}=\left(\begin{array}{ll}
1 & 0 \\
0 & \varepsilon
\end{array}\right)
$$

которые порождают в Aut $\mathrm{M}_{2}$ подгруппу, изоморфную знакопеременной группе $A_{4}$. Следовательно, Aut $\mathscr{E}_{2} \cong A_{4}$.

3) Из канонического подстановочного представления степени 4 симметрической групшы $S_{4}$, полагая $H_{i}=G_{i} \cong S_{3}$, получаем сбалансированную $3-(4,3)$ систему идемпотентов $\mathscr{E}_{3}$ в $\mathrm{M}_{3}$ :

$$
\begin{aligned}
& f_{1}=\frac{1}{3}\left(\begin{array}{rrr}
1 & 1 & 1 \\
1 & 1 & 1 \\
1 & 1 & 1
\end{array}\right), f_{2}=\frac{1}{3}\left(\begin{array}{rrr}
1 & -1 & 1 \\
-1 & 1 & -1 \\
1 & -1 & 1
\end{array}\right) \\
& f_{3}=\frac{1}{3}\left(\begin{array}{rrr}
-1 & -1 \\
-1 & 1 & 1 \\
-1 & 1 & 1
\end{array}\right), f_{4}=\frac{1}{3}\left(\begin{array}{rrr}
1 & 1 & -1 \\
1 & 1 & -1 \\
-1 & -1 & 1
\end{array}\right) .
\end{aligned}
$$

Очевидно, Aut $\mathscr{E}_{3} \cong S_{4}$.

4) Рассмотрим знакопеременную группу $A_{5}$ и ее 2-транзитивное представление степени 6 . В качестве подгрупп $H_{i}$ возьмем семейство силовских 5-подгрупп. Используя линейное представление $A_{5}$ степени 3 (любое из двух), получим 2-(6,3) систему идемпотентов $\mathscr{E}_{4}$ в $\mathrm{M}_{3}$. Можно показать, что Aut $\mathscr{E}_{4} \cong A_{5}$. 


\section{Список литературы}

1. Иванов Д. Н. Ортогоналњные разложения полупростых ассоциативных алгебр // Вестн. МГУ. Сер. 1. Матем., мех. 1988. № 1. С. 9-14.

2. Иванов Д. Н. Теорема о подалгебрах, образующих ортогональное разложение ассоциативной алгебры // УМН. 1989. Т. 44. № 2. С. 231-232.

3. Иванов Д. Н. Однородные ортогональные разложения коммутативных алгебр и матрицы Адамара // Фундамент. и прикл. матем. 1995. Т. 1. № 4. С. 1107-1110.

4. Холл М. Комбинаторика. М.: Мир, 1970.

5. Кострикин А.И., Кострикин И. А., Уфнаровский В. А. Ортогональные разложения простых алгебр Ли (тип $\left.A_{n}\right)$ // Труды МИАН. 1981. Т. 158. С. 105-120.

6. Харари Ф. Теория графов. М.: Мир, 1973.

7. Kostrikin A.I., Pham Huu Tiep. Orthogonal decompositions and integral lattices. Berlin: Walter de Gruyter, 1994

8. Камерон П., ван Линт Джс. Теория графов, теория кодирования и блок-схемы. М.: Наука, 1980.

Тверской государственньй университет

Поступила в редакцию

E-mail: Dmitry.Ivanov@tversu.ru

26.02.1998 